\title{
Constraints on Self Interacting Dark Matter from IceCube Results
}

\author{
Ivone F. M. Albuquerque, ${ }^{a}$ Carlos Pérez de los $\operatorname{Heros}^{b}$ and Denis \\ S. Robertson ${ }^{a}$ \\ ${ }^{a}$ Instituto de Física, Universidade de São Paulo, São Paulo, Brazil \\ ${ }^{b}$ Department of Physics and Astronomy. Uppsala University. Uppsala. Sweden \\ E-mail: ifreire@if.usp.br, denistefanrs@gmail.com,cph@physics.uu.se
}

\begin{abstract}
If dark matter particles self-interact, their capture by astrophysical objects should be enhanced. As a consequence, the rate by which they annihilate at the center of the object will increase. If their self scattering is strong, it can be observed indirectly through an enhancement of the flux of their annihilation products. Here we investigate the effect of self-interaction on the neutrino flux produced by annihilating dark matter in the center of the Sun. We consider annihilation into two channels: $W^{+} W^{-}$(or $\tau^{+} \tau^{-}$for a dark matter mass below the $W$ mass) and $b \bar{b}$. We estimate the event rate in the IceCube detector, using its 79-string configuration, and compare our prediction to their experimental results, hence probing dark matter self interacting models.
\end{abstract}

Keywords: Dark matter, Neutrinos, Neutrino Telscopes 


\section{Contents}

1 Introduction 1

2 Dark Matter Capture and Annihilation Rates Enhancement due to SelfInteractions

$3 \quad$ Neutrino Flux From SIDM Models 4

4 Probing SIDM Models $\quad 6$

$\begin{array}{llr}5 & \text { Conclusions } & 9\end{array}$

\section{Introduction}

Current cosmological observations indicate that our Universe is flat, and composed mainly by dark matter and dark energy. Observations at large scales are very well fit by collisionless cold dark matter models (CDM). However these models present potential problems related to small scale structure formation, one of them being referred to as the core/cusp problem [1]. While structure formation simulations [2-4], based on CDM models, present a steep cusp density profile, observations of dwarf galaxies [5-9] indicate a cored density profile rather than a cusped one. Also CDM simulations evolve to very dense subhaloes of Milky Way type galaxies, which is a problem since these cannot host its brightest satellites $[10,11]$. This discrepancy is known as the "too big to fail problem", given that it would be hard to miss the observation of these substructures.

Warm dark matter models (WDM) have been proposed as a solution to these inconsistencies, since it was expected that they should develop shallower density profiles at small radius, and would avoid unreasonably dense subhaloes [12]. However there are also discrepancies between simulations and observations, where the core/cusp problem is not solved by WDM [13], and thermal WDM candidates seem to be ruled out by Lyman- $\alpha$ forest results [14].

Another very interesting solution to these small scale problems is to consider dark matter self-interactions (SIDM) [15], where dark matter particles scatter among themselves, instead of collisionless CDM models. If this scatter is strong enough, the halo central density profile will be softened in relation to a pure CDM model. Cosmological simulations [16, 17] show that SIDM models with a ratio between the self-interaction cross sections over the dark matter mass, $\sigma_{\chi \chi} / m_{\chi} \sim \vartheta\left(0.1 \mathrm{~cm}^{2} / \mathrm{g}\right)$ will reconcile simulations with the observed dwarf galaxies properties, while these self-interactions will not modify the CDM behaviour at large scale.

It should be noticed however that the small scale potential problems might not be associated with the CDM models themselves, but with other structure evolution features. As examples, the "too big to fail problem" can be solved by the inclusion of baryons in structure formation simulations which changes the shape of dark matter profiles [18], or by the fact that similar host halos present variations in their subhalos properties [19]. The inclusion of baryons, as well as three dimensional mass distributions [20] might also contribute to cored profiles [21]. Although these solutions might bring CDM simulations to agree with observations, the SIDM solution should also be contemplated and seriously explored. 
In this paper we probe SIDM models through the neutrino flux produced from dark matter annihilation in the center of the Sun. Dark matter scattering off the dark matter that has already been captured in the Sun's potential well will enhance its capture rate, and consequently its annihilation rate. In this way, if dark matter self-interacts in the Sun, the neutrino flux should be enhanced when compared to the one produced by collisionless dark matter annihilation. This was noted in [22].

Note that, in scenarios where the $\sigma_{\chi \chi}$ is velocity-independent, the annihilation process by itself does not differ between collisionless CDM and SIDM. The enhancement comes exclusively through the capture rate. Not only there is an increase in the number of dark matter particles that are captured, but as the equilibrium between capture and annihilation happens faster when considering self-interactions, the maximum annihilation rate is reached earlier than in pure CDM models.

The most robust constraint to SIDM comes from an analysis of the Bullet Cluster matter distribution [24], which excludes $\sigma_{\chi \chi} / m_{\chi}>1.25 \mathrm{~cm}^{2} / \mathrm{g}$. There are also constraints from an analysis on the core densities of galaxy clusters, low mass spiral galaxies and dwarf spheroidal galaxies [16], and from halo shapes [17], excluding $\sigma_{\chi \chi} / m_{\chi}>1.0 \mathrm{~cm}^{2} / \mathrm{g}$. Another interesting analysis, based on the kinematics of dwarf spheroidals [25], estimates that SIDM will only alleviate CDM small scale problems when $\sigma_{\chi \chi} / m_{\chi}>0.1 \mathrm{~cm}^{2} / \mathrm{g}$. These analyses are more assumption dependent than the Bullet Cluster analysis. If we take all these limits as robust, and consider the area where SIDM is expected to be effective, there is still a small, but nonthe-less interesting, non probed region between $\left(1.0>\sigma_{\chi \chi} / m_{\chi}>0.1\right) \mathrm{cm}^{2} / \mathrm{g}$. Our analysis will probe most of this allowed region. At the same time it will, in an independent way, probe the parameter space region excluded by the Bullet Cluster and halo shapes analyses.

We note that these analyses imply that only very strong $\sigma_{\chi \chi}$, at the $\vartheta\left(10^{-22} \mathrm{~cm}^{2}\right)$, can solve CDM potential cosmological problems. Although these are stronger than cross sections for nucleon-nucleon interactions, and not at first hand expected, it is important to probe these allowed regions of SIDM.

Our investigation proceeds by computing the neutrino flux from dark matter annihilations in the Sun through Monte Carlo simulations, and we consider two extreme cases as benchmarks: annihilation into $W^{+} W^{-}$(or $\tau^{+} \tau^{-}$when the dark matter mass is less than the $W$ mass) and $b \bar{b}$. We determine the expected neutrino flux at the IceCube detector, and based on the fact that there was no measured anomalous neutrino flux from the Sun [23] we set limits on the $\left(\sigma_{\chi \chi}, m_{\chi}\right)$ parameter space.

In the next section we discuss the dark matter capture and annihilation rates enhancement due to self-interactions. We then describe how we determine the neutrino rate in IceCube. Following we analyze our results, and compare our predictions to IceCube results and finally describe our conclusions.

\section{Dark Matter Capture and Annihilation Rates Enhancement due to Self-Interactions}

If dark matter self interacts, the evolution of its number $\left(N_{\chi}\right)$ in the Sun will follow

$$
\dot{N}_{\chi}=\Gamma_{C}+\Gamma_{\chi \chi}-\Gamma_{A},
$$

where $\Gamma_{C}$ and $\Gamma_{\chi \chi}$ are the capture rates for dark matter particles that interact with the Sun's nuclei, and due to self-interactions, respectively. $\Gamma_{A}$ is the annihilation rate, which equals $N_{\chi}^{2} C_{A}$, where $C_{A}$ depends on the dark matter distribution in the Sun [26, 27], and 
is given by $C_{A} \equiv\left\langle\sigma_{A} v\right\rangle / V_{\text {eff }}$, where $\left\langle\sigma_{A} v\right\rangle$ is the relative velocity averaged annihilation cross section. $V_{\text {eff }}$ represents the dark matter effective volume at the center of the Sun. Assuming an isothermal distribution, and taking the Sun's temperature as $1.57 \times 10^{7} \mathrm{~K}$ [28], $V_{\text {eff }}=6.9 \times 10^{27} \times\left(100 \mathrm{GeV} / m_{\chi}\right)^{3 / 2} \mathrm{~cm}^{3}$.

The dark matter capture rate due to elastic scatter off the Sun's nuclei is given by $[26,27]$

$\Gamma_{C}^{S I}=6.8 \times 10^{20} \mathrm{~s}^{-1} \frac{n_{\chi}}{0.4 \mathrm{~cm}^{-3}} \frac{\sigma_{\chi n}}{10^{-44} \mathrm{~cm}^{2}} \frac{270 \mathrm{~km} / \mathrm{s}}{\bar{v}} \sum F_{i}\left(m_{\chi}\right) A_{i}^{3}\left(\frac{m_{\chi}+m_{p}}{m_{\chi}+m_{N_{i}}}\right)^{2} \delta_{i} K\left(\frac{m_{\chi}}{m_{N_{i}}}\right)^{2}$

and depends on the dark matter local number density $n_{\chi}$, mass, velocity dispersion $\bar{v}$, spin independent dark matter nucleon cross section $\sigma_{\chi n}$, and the sum is over all Sun's nuclear species i. $F_{i}\left(m_{\chi}\right)$ accounts for the interaction form factor suppression, $\delta_{i}$ for the mass fraction and distribution of the various nuclei over the Sun, and $K\left(\frac{m_{\chi}}{m_{N_{i}}}\right)$ is a kinematic suppression factor. $m_{N_{i}}$ is the nucleus $i$ mass and $A_{i}$ its atomic mass. The cross sections for dark matternuclei interaction $\sigma_{\chi N_{i}}$ relate to $\sigma_{\chi n}$ as $\sigma_{\chi N_{i}}=\sigma_{\chi n} A_{i}^{2}\left(\frac{\mu_{\chi N_{i}}}{\mu_{\chi p}}\right)^{2}$, where $\mu$ is the reduced mass, and we approximated $m_{N_{i}} \sim A_{i} m_{p}$, where $m_{p}$ is the proton's mass and is not distinguished from a neutron, in a better than $1 \%$ approximation. To compute $\delta_{i}$ we take the nucleus mass fraction as given in [29] and its distribution from [27]. The distribution can be conveniently represented by a dimensionless gravitational potential $v_{\text {esc }}^{2}(r) / v_{\text {esc }}^{2}\left(R_{\odot}\right)$ as described in [26].

When computing the capture rate due to self-interactions, all terms in the sum of the above equation have to account for self instead of $\chi$-nuclei interactions. The number of captured dark matter particles increases with time, until the capture rate reaches equilibrium with the annihilation rate. The self-interaction capture rate is given by [22] :

$$
\Gamma_{\chi \chi}=N_{\chi} \sqrt{\frac{3}{2}} n_{\chi} \sigma_{\chi \chi} \frac{v_{e s c}^{2}\left(R_{\odot}\right)}{\bar{v}}\left\langle\phi_{\chi}\right\rangle \frac{\operatorname{erf}(\eta)}{\eta}
$$

where $\left\langle\phi_{\chi}\right\rangle$ accounts for the dark matter distribution, which for the Sun is approximately 5.1 [32] since it concentrates more towards the center, $v_{e s c}\left(R_{\odot}\right)=617.5 \mathrm{~km} / \mathrm{s}$ is the Sun's escape velocity at its surface, and $\eta=\sqrt{\frac{3}{2}} \frac{v_{\odot}}{\bar{v}}$, where $v_{\odot}=220 \mathrm{~km} / \mathrm{s}$ is the Sun velocity through the dark matter halo, which we assume has a Maxwell-Boltzman distribution with a velocity dispersion of $\bar{v}=270 \mathrm{~km} / \mathrm{s}$. We take the local dark matter density as $\rho_{\chi}=0.4 \mathrm{GeV} / \mathrm{cm}^{3}$ $[30,31]$. It is useful to define $\Gamma_{\chi \chi}^{\prime}=\Gamma / N_{\chi}$, noting that it is independent of $N_{\chi}$.

An important effect of SIDM is that the timescale for capture and annihilation equilibrium, given by

$$
\tau_{\chi \chi}=\left(\Gamma_{C} C_{A}+\frac{\Gamma_{\chi \chi}^{\prime 2}}{4}\right)^{-1 / 2},
$$

is shorter than for CDM-only models. This effect can be seen in Figure 1, where we compare the time evolution of the number of collisionless dark matter particles (CDM models) to $\mathrm{CDM}+\mathrm{SIDM}$ (SIDM) models. As in the CDM case, once the equilibrium is reached, the annihilation rate is maximum and the number of dark matter particles in the Sun is stable. The stronger $\sigma_{\chi \chi}$ is, the faster the equilibrium will be reached. For $\sigma_{\chi \chi}=3 \times 10^{-22} \mathrm{~cm}^{2}$ and a low $\sigma_{\chi n}$ the equilibrium will only have been reached in the Sun if SIDM is considered.

Figure 1 also shows that SIDM models with strong $\sigma_{\chi \chi} \sim \vartheta\left(10^{-22} \mathrm{~cm}^{2}\right)$, and in the $\sigma_{\chi n}$ region which is not excluded by direct detection experiments, will enhance the flux of dark 

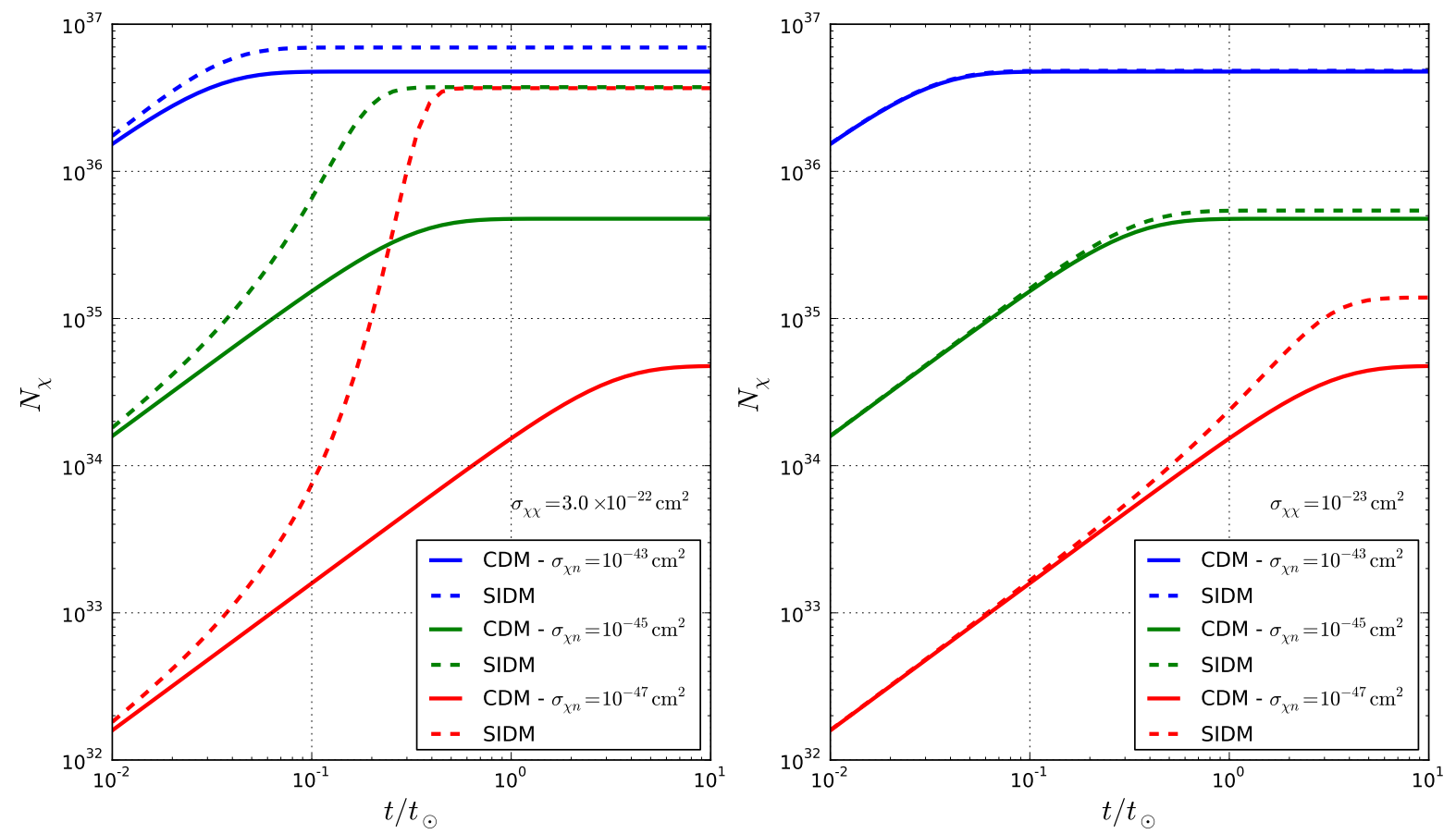

Figure 1. Time evolution of dark matter particles in the Sun. On the left $\sigma_{\chi \chi}=3 \times 10^{-22} \mathrm{~cm}^{2}$ and on the right $\sigma_{\chi \chi}=10^{-23} \mathrm{~cm}^{2}$. Solid curves are for CDM models while dashed curves for CDM+SIDM. Curves are for different $\sigma_{\chi n}$ values as labelled.

matter annihilation products from the center of the Sun. This enhancement is significant when compared to pure CDM models. One interesting feature to be observed [22] when determining the neutrino flux from dark matter annihilation, is that when $\sigma_{\chi \chi}$ is strong enough it will become independent of $\sigma_{\chi n}$. This can be seen in the left plot of Figure 1, through the convergence of the red and green lines.

The Sun's spin-dependent (SD) capture rate will mainly include interactions with hydrogen. In this case there is no significant form factor suppression, and by modifying equation 2.2 accordingly, the spin-dependent rate is given by:

$$
\Gamma_{C}^{S D}=17.3 \times 10^{20} \mathrm{~s}^{-1} \frac{n_{\chi}}{0.4 \mathrm{~cm}^{-3}} \frac{270 \mathrm{~km} / \mathrm{s}}{\bar{v}} \frac{\sigma_{\chi H}}{10^{-44} \mathrm{~cm}^{2}} \times K\left(\frac{m_{\chi}}{m_{H}}\right)
$$

The solution for the dark matter number evolution equation (Equation 2.1) is given by

$$
N_{\chi}=\frac{\Gamma_{C} \tanh \left(t / \tau_{\chi \chi}\right)}{\tau_{\chi \chi}^{-1}-\Gamma_{\chi \chi}^{\prime} \tanh \left(t / \tau_{\chi \chi}\right) / 2}
$$

and allows us to determine the annihilation rate $\Gamma_{A}=C_{A} N_{\chi}^{2} / 2$.

\section{$3 \quad$ Neutrino Flux From SIDM Models}

In order to determine the neutrino flux from dark matter annihilation in the center of the Sun, we perform Monte Carlo simulations using the WimpSim package [33]. We simulate a generic dark matter particle $\chi$ and antiparticle $\bar{\chi}$ annihilating into $W^{+} W^{-}$(or into $\tau^{+} \tau^{-}$for $m_{\chi}<$ $m_{W}$ ), and to $b \bar{b}$. These channels were chosen since their decay chain will produce neutrinos within a wide energy range. They are also the ones analyzed by the IceCube collaboration, 


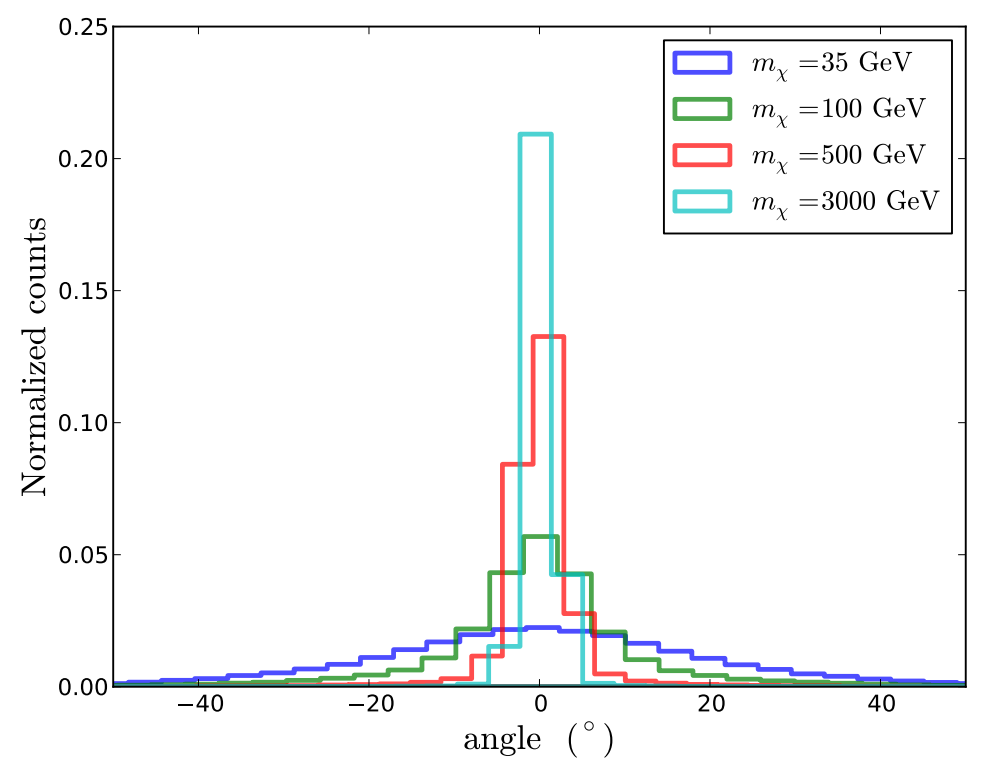

Figure 2. Angular smearing about the Sun-IceCube axis due to the experimental angular resolution [35], for different dark matter masses.

and therefore allow us to compare our results to theirs. The $W^{+} W^{-}$and $b \bar{b}$ decay chain will produce neutrinos, either as primaries or secondaries, which will be propagated to the position of the IceCube detector at the Earth. As a result of our simulation, two neutrino energy spectra $\frac{d \Phi_{\nu}}{d E}$ at the detector are generated, one for $W^{+} W^{-} / \tau^{+} \tau^{-}$channel and the other for $b \bar{b}$. We simulate two sets of events, one corresponding to a winter and the other to a summer period. We follow the same definition of data sets as IceCube, where the winter is further split into two sets, one composed by low energy events, with neutrino energies $E_{\nu} \lesssim 95 \mathrm{GeV}$ (WL), and the other to high energy events (WH). The summer set (SL) includes only low energy events, and their observation requires that the neutrino interaction occurs inside the DeepCore [36] detector, which is embedded in IceCube. This requirement rejects down-going atmospheric muons which traverse the detector, faking a possible signal.

Neutrino oscillations as well as charge and neutral current interactions are considered, and we assume the standard parameters for neutrino oscillations [34]. This latter effect will be significant for neutrinos travelling from the Sun to the Earth, and the initial flavor composition will differ from the one near the detector. In this analysis we consider only muon neutrinos arriving at IceCube.

In order to compare our predictions to observations, we need to account for IceCube's experimental angular resolution, which is energy dependent and given in [35] . The average angular error is about $4^{o}$ for $100 \mathrm{GeV}$ neutrinos, increasing (decreasing) for lower (higher) energies. We include this reconstruction effect by smearing the arrival direction of each simulated event by a gaussian distribution with its $\sigma$ equal to the experimental angular resolution. Figure 2 shows the arrival angular distributions about the Sun-IceCube axis, for different dark matter mass values. We then remove all events with a smearing angular direction $\theta>3^{\circ}$, which is IceCube's accepted angular direction for events coming from the Sun [23]. Figure 3 shows the event reduction due to the angular requirement for the 

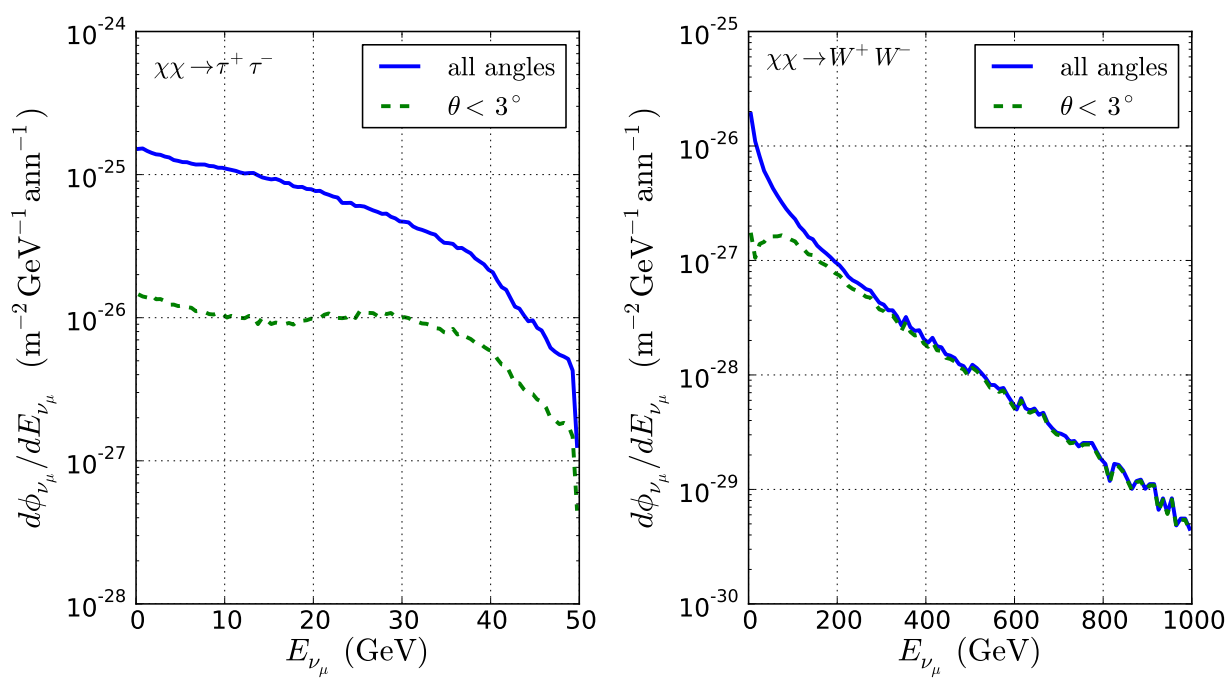

Figure 3. Neutrino flux versus neutrino energy for $m_{\chi}=50 \mathrm{GeV}$ and $1000 \mathrm{GeV}$ annihilating into $W^{+} W^{-} / \tau^{+} \tau^{-}$. The blue solid line includes all simulated events and the green dashed line events with smeared angular direction $\theta \lesssim 3^{\circ}$.

$W^{+} W^{-} / \tau^{+} \tau^{-}$channel, for a low $(50 \mathrm{GeV})$ and large $(1000 \mathrm{GeV})$ dark matter mass.

The expected number of muon neutrinos $N_{\nu_{\mu}}$, from dark matter annihilation in the Sun, in IceCube will be given by

$$
N_{\nu_{\mu}}=\Gamma_{A} t_{\exp } \int_{E_{\mathrm{thr}}} \frac{d \Phi_{\nu_{\mu}}}{d E} A_{\mathrm{eff}}(E) \mathrm{d} E,
$$

where $t_{\exp }$ is the exposure time and depends on which data set is analyzed, being 150 days for the winter and 167 days for the summer period. $A_{\text {eff }}$ is IceCube's effective area, which accounts not only for the energy dependent trigger and analysis efficiencies, but also for the neutrino-nucleon interaction probability, and the converted muon energy loss before detection. We take $A_{\text {eff }}$ as given as a function of the neutrino energy for each data set in [35]. Once we have our prediction for the integrated number of events in IceCube, we can compare it with the experimental result. Figure 4 exemplifies the predicted spectrum of muons arriving at IceCube, from $800 \mathrm{GeV}$ dark matter annihilation into $W^{+} W^{-}$, for different values of $\sigma_{\chi \chi} / m_{\chi}$, and $\sigma_{\chi n}=1.0 \times 10^{-44} \mathrm{~cm}^{2}$ as a function of the neutrino energy. The enhancement on the expected number of events due to self-interactions is clear in this figure.

\section{$4 \quad$ Probing SIDM Models}

The IceCube collaboration has searched for signals from dark matter annihilation in the Sun with its 79-string telescope's configuration [23]. It covered a large neutrino energy range. By including detection with the DeepCore array [36], which is embedded in IceCube, they lowered their energy threshold down to $10 \mathrm{GeV}$. IceCube's digital optical modules that surround this array work as a background veto, allowing to discriminate muons produced within the infill, and accumulate useful data during the summer period. 


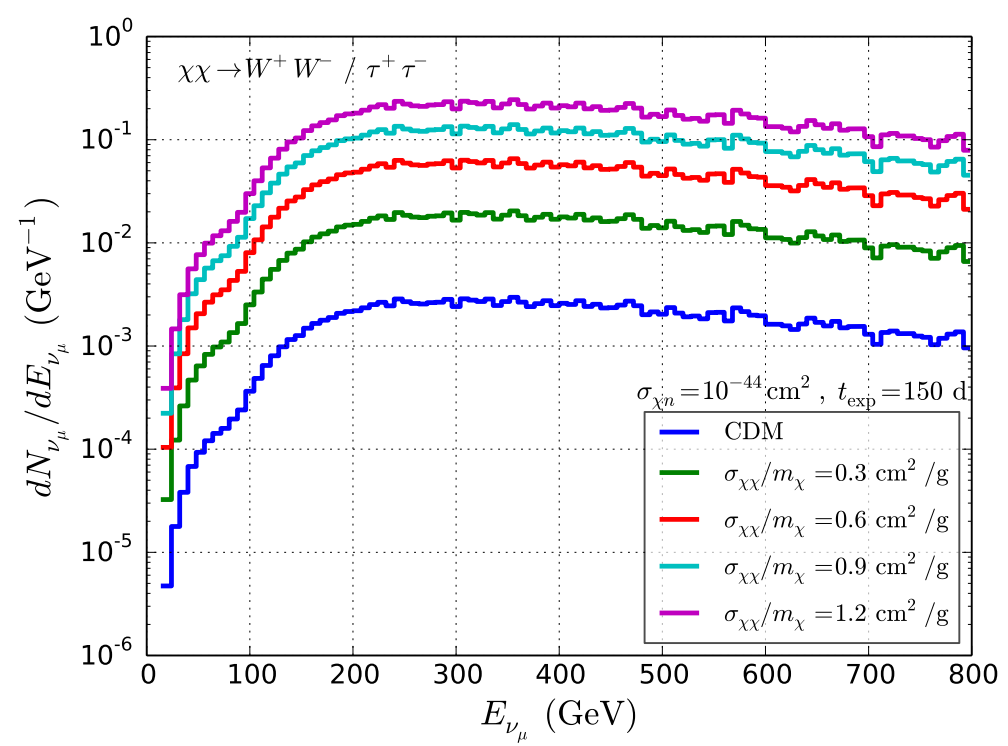

Figure 4. Muon differential energy spectrum at the detector as a function of the neutrino energy, assuming CDM or CDM+SIDM models, for different $\sigma_{\chi \chi} / m_{\chi}$ values as labelled. The integrated number of events is 1.4 for CDM models, $9.6(30.8,66.0,115.2)$ for $\sigma_{\chi \chi} / m_{\chi}=0.3(0.6,0.9,1.2) \mathrm{cm}^{2} / \mathrm{g}$. Results are for $m_{\chi}=800 \mathrm{GeV}$ annihilating into the $W^{+} W^{-}$channel.

Their main background consists of atmospheric muons and neutrinos. As described in Section 3 they have three separated data sets, two for the winter period (WL and WH, where $\mathrm{L}$ and $\mathrm{H}$ stand for low and high energies), reflecting the different neutrino energies covered by both channels analyzed, and one for the low energy events collected during the summer period (SL). Each of these data samples, as well as each of the annihilation channels, were analyzed in different ways given that their background have different characteristics. IceCube concludes that their data is consistent with the expected background and impose limits on both WIMP spin-independent and spin-dependent models [23].

IceCube results are expressed as an upper limit on the number of signal events at $90 \%$ CL $\left(\mu_{90}\right)$, as a function of the dark matter mass and annihilation channel. Any model that predicts a larger number of events during the data taking period can be ruled out. We use this limit to compare our predictions and to probe various SIDM models. Our analysis probes $\sigma_{\chi \chi}$ as a function of $m_{\chi}$. Figure 5 shows the regions that are excluded at $90 \%$ CL by our spin-independent $W^{+} W^{-} / \tau^{+} \tau^{-}$channel analysis, where each plot shows limits assuming two values for the thermal annihilation cross section $\left(0.1\right.$ and $\left.3 \times 10^{-26} \mathrm{~cm}^{3} / \mathrm{s}\right)$. As can be seen in this figure, a large fraction of the previously allowed region of the $\sigma_{\chi \chi}$ versus $m_{\chi}$ parameter space is excluded by our analysis. The smaller (larger) the $\left\langle\sigma_{A} v\right\rangle\left(\sigma_{\chi n}\right)$ the larger the excluded region.

The limits shown in this figure do not change significantly for different $\sigma_{\chi n}$ values, as seen from the four different plots. This is consistent with the fact, mentioned when discussing figure 1, that for strong $\sigma_{\chi \chi}$ values, the neutrino flux is independent of $\sigma_{\chi n}$.

Figure 5 also shows the regions excluded by analyses of the Bullet Cluster [24] and the halo shapes [17], which were briefly discussed in the introduction. The region estimated by 

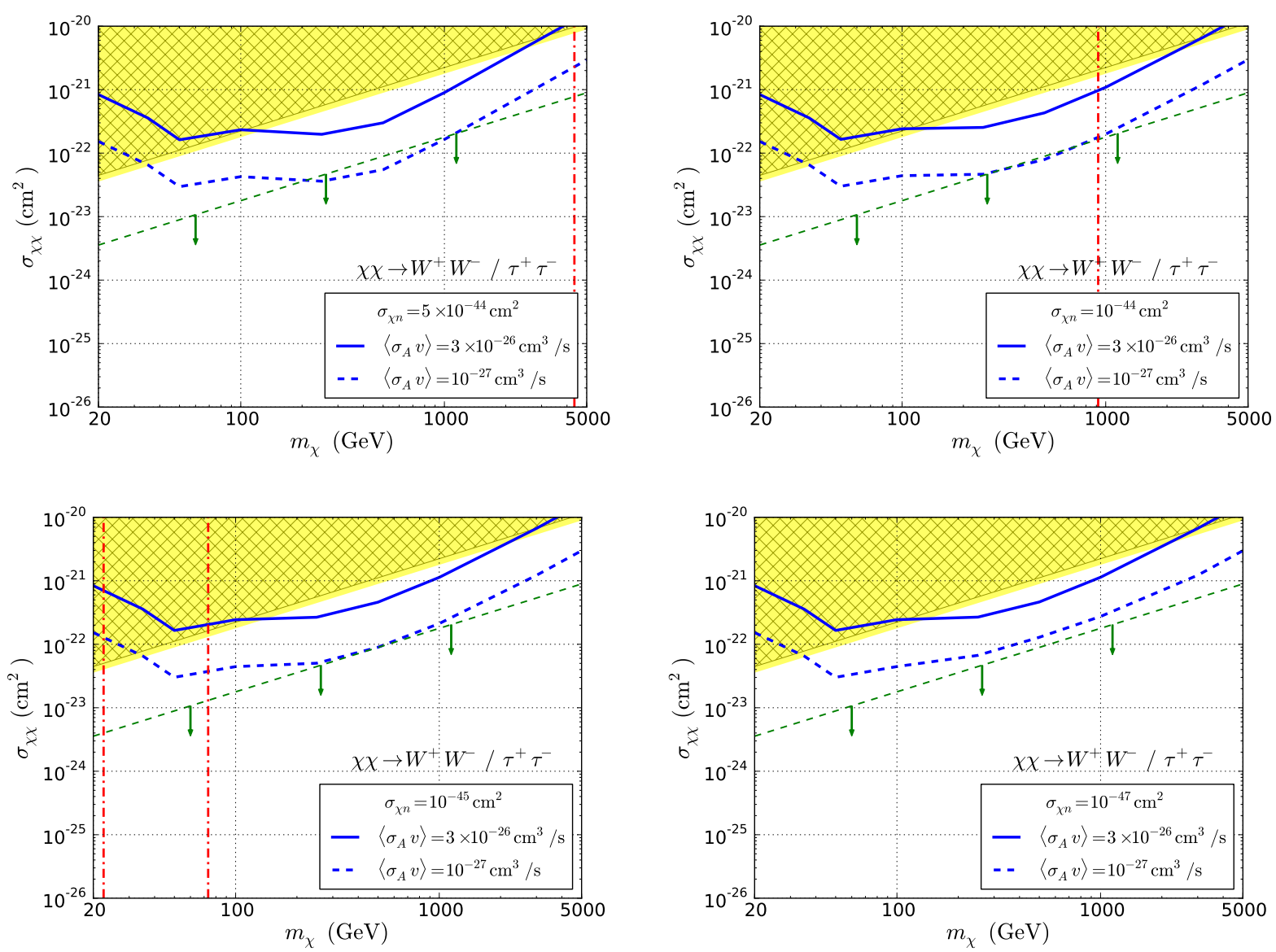

Figure 5. Self annihilation cross section $\sigma_{\chi \chi}$ versus dark matter mass. The regions above the blue curves exclude models with annihilation into $W^{+} W^{-} / \tau^{+} \tau^{-}$at $90 \%$ CL by our analysis. The solid (dashed) line is for a thermal annihilation cross section $\left\langle\sigma_{A} v\right\rangle=3(0.1) \times 10^{-26} \mathrm{~cm}^{3} / \mathrm{s}$. Each plot considers a different $\sigma_{\chi n}$ value, as labeled. Exclusion regions from a Bullet Cluster analysis [24] is shown in black hatches, and by halo shapes [17] in yellow. The region below the dashed green line, shows the region were SIDM is too weak too alleviate CDM potential problems, based on the dwarf spheroidals analysis [25]. The red lines show the direct detection limits from LUX [37], where either the region to the left or between the lines are excluded. LUX results do not probe $\sigma_{\chi n} \lesssim 10^{-47} \mathrm{~cm}^{2}$, which is represented in the bottom right plot.

the dwarf spheroidals analysis [25] as being too weak to solve CDM potential problems falls below the green line. Also the region excluded by the direct detection LUX [37] collaboration is shown, noting that our analysis excludes independently SIDM models that fall on the right hand side of the red line and above the blue lines. LUX does not probe scenarios with $\sigma_{\chi n} \lesssim 7 \times 10^{-46} \mathrm{~cm}^{2}$.

The $b \bar{b}$ channel results are shown in Figure 6 . As this channel is softer and produces lower energy neutrinos, the exclusion regions are smaller than the ones for the $W^{+} W^{-} / \tau^{+} \tau^{-}$ channel. If this annihilation channel holds, it confirms independently most of the region excluded by the Bullet Cluster and halo shape analyses.

We have also estimated the event rate for spin-dependent dark matter interactions, which mainly consists of dark matter interacting with hydrogen in the Sun. For the channels we are analyzing, IceCube has the most stringent limits on the spin-dependent WIMP-proton cross section [23]. We proceed in the same way as for spin-independent scattering. Equation 3.1 depends on the annihilation rate which in its turn depends on the capture rate. For spin-dependent interactions the latter is given by equation 2.5 instead of equation 2.2. Our 

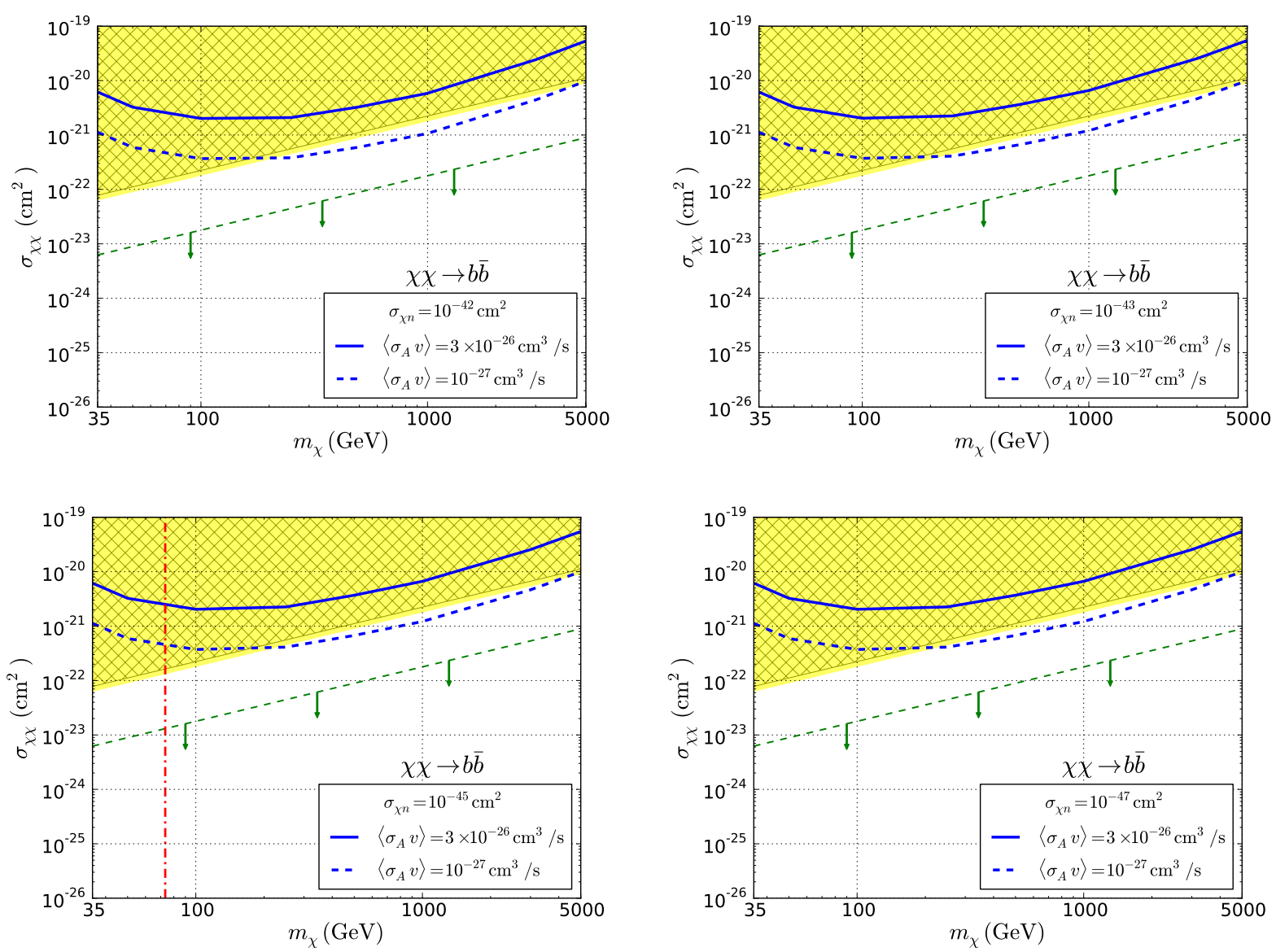

Figure 6. Same as previous figure but now for dark matter annihilation into $b \bar{b}$. The regions above the blue curves are excluded at $90 \%$ CL by our analysis. The solid (dashed) line is for a thermal annihilation cross section $\left\langle\sigma_{A} v\right\rangle=3(0.1) \times 10^{-26} \mathrm{~cm}^{3} / \mathrm{s}$. Each plot considers a different $\sigma_{\chi n}$ value, as labeled. In these plots LUX [37] direct detection results exclude all the regions shown on the top figures, the region to the left of the red lines in the leftmost bottom plot, and does not probe the rightmost bottom plot region.

results for the $W^{+} W^{-}$annihilation channel are shown in Figure 7 and for the $b \bar{b}$ channel in Figure 8. We choose $\sigma_{\chi H}$ values that are not constrained by IceCube [23].

Our spin-dependent exclusion region is larger than the spin independent, which is expected since we assume larger cross sections than the ones for the spin-independent analysis. As for the spin-independent, the exclusion region for the $b \bar{b}$ is as expected smaller than the one for the $W^{+} W^{-} / \tau^{+} \tau^{-}$channel.

\section{Conclusions}

We have demonstrated that most SIDM models with strong self-interacting cross sections, at $\sigma_{\chi \chi} \gtrsim \vartheta\left(10^{-22}\right) \mathrm{cm}^{2}$ (or $\left.\vartheta\left(10^{-23}\right)\right)$ for $\left\langle\sigma_{A} v\right\rangle=3 \times 10^{-26} \mathrm{~cm}^{3} / \mathrm{s}\left(1 \times 10^{-27} \mathrm{~cm}^{3} / \mathrm{s}\right.$ ), are ruled out if they annihilate into $W^{+} W^{-}$s. This exclusion comes from the comparison of our predicted neutrino signal in the IceCube detector to their observations [23]. This result is valid for both spin-dependent and independent interactions, with the first one being more stringent. Our results are summarized in figures 5 to 8 .

If the assumptions made in the analyses presented in [17, 24] and [25] are correct, and dark matter annihilates mainly into the $W^{+} W^{-} / \tau^{+} \tau^{-}$channel, most of the significant SIDM scenarios are excluded for thermal annihilation cross sections $\left\langle\sigma_{A} v\right\rangle \leq 10^{-27} \mathrm{~cm}^{2}$. 

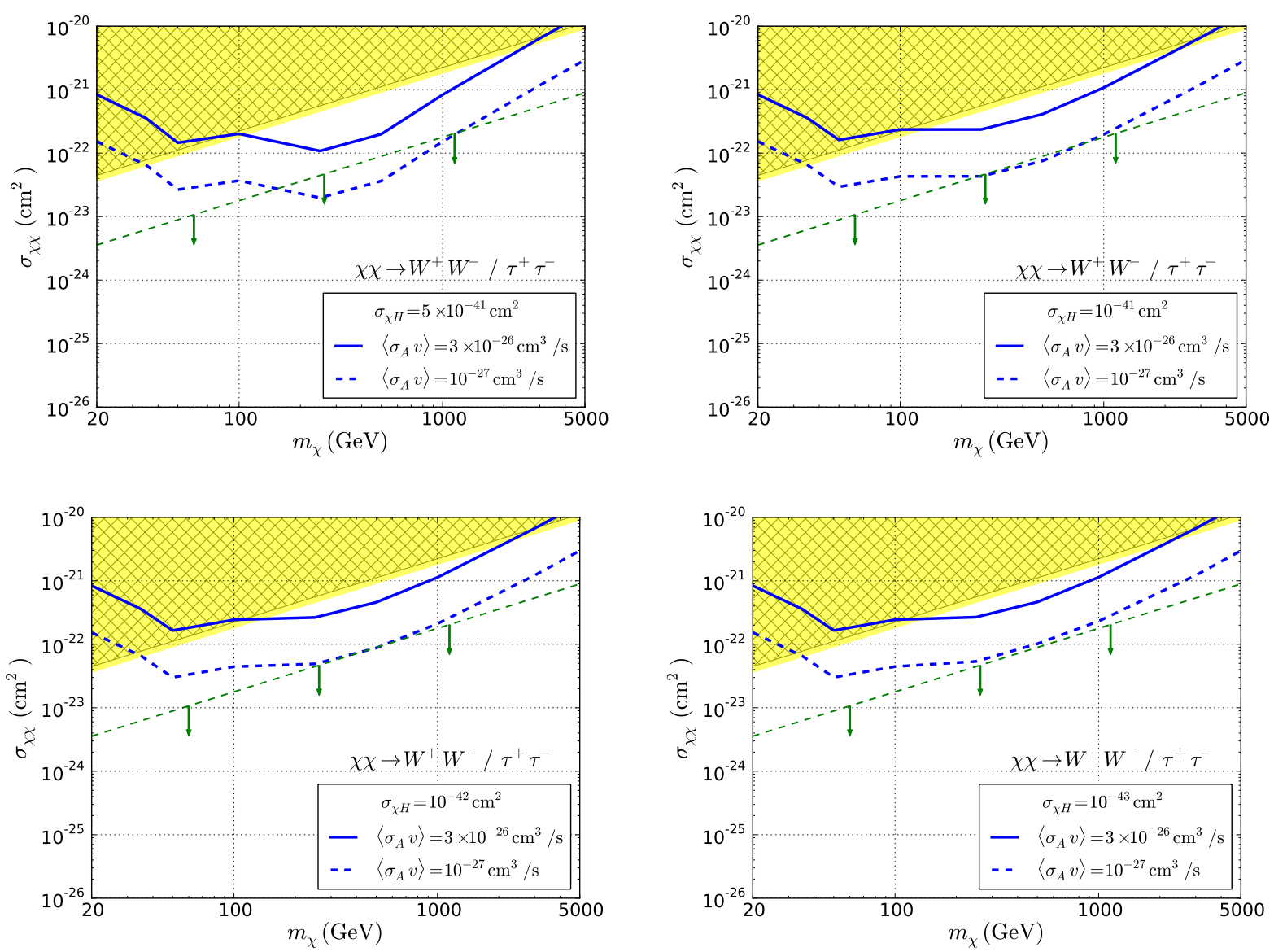

Figure 7. Same as Figure 5 but now considering $\sigma_{\chi H}$ spin-dependent interactions. There are no spin-dependent direct detection probes of the region shown in these plots.

Under these assumptions, all self-interacting models with $300 \lesssim m_{\chi} \lesssim 1000 \mathrm{GeV}$, and in the region identified by the dwarf spheroidals analysis as the one which would alleviate the CDM small scale potential problems, are now excluded. In this case, solutions to these problems will have to be encountered in different SIDM scenarios, where, for example, the annihilation channel produces lower energy neutrinos, as for instance the $b \bar{b}$ channel. Another possibility is to consider that the self scattering $\sigma_{\chi \chi}$ is velocity dependent [38]. In our analysis, we determine the self scattering rate by considering a velocity independent $\sigma_{\chi \chi}$, as can be seen from equation 2.3. It is interesting to check the modifications to our results from a velocity dependent analysis [39].

In relation to the $b \bar{b}$ channel, we independently confirm the Bullet cluster [24] and halo shapes [17] analyses. For $\left\langle\sigma_{A} v\right\rangle \leq 10^{-27} \mathrm{~cm}^{2}$ a large region of strong $\sigma_{\chi \chi}$ is ruled out, and for $\left\langle\sigma_{A} v\right\rangle=3 \times 10^{-26} \mathrm{~cm}^{2}$ most of the $\sigma_{\chi \chi} \gtrsim 5 \times 10^{-21} \mathrm{~cm}^{2}$ region is excluded.

We also compared our results to the most stringent direct detection results, from the LUX [37] collaboration. As can be seen from Figures 5 and 6, direct detection experiments have not yet probed the region with $\sigma_{\chi n} \lesssim 10^{-45} \mathrm{~cm}^{2}$ which is probed by our analysis.

\section{Acknowledgments}

IA acknowledges the partial support from the Brazilian National Counsel for Scientific Research (CNPq), and from the European Union FP7 ITN INVISIBLES (Marie Curie Actions, 

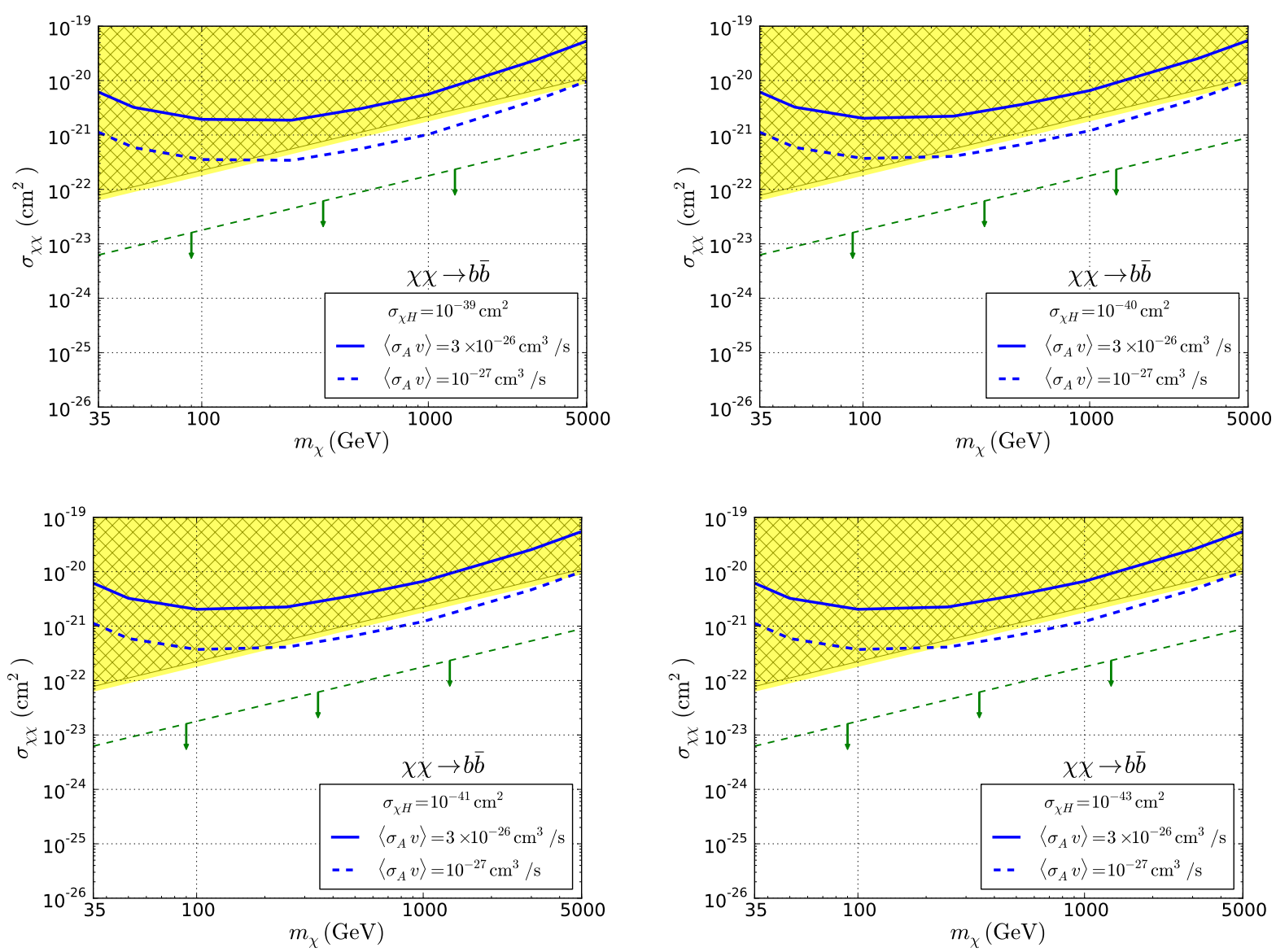

Figure 8. Same as Figure 6 but now considering $\sigma_{\chi H}$ spin-dependent interactions.

PITN- GA-2011- 289442). DSR was funded by the State of São Paulo Research Foundation (FAPESP). CPH is partially supported by a grant from the Swedish Research Council.

\section{References}

[1] W. J. G. de Blok, Adv. Astron. 2010, 789293 (2010).

[2] B. Moore, Nature 370, 629 (1994).

[3] R. A. Flores and J. R. Primack, Astrophys. J. 427, L1 (1994).

[4] J. F. Navarro, C. S. Frenk and S. D. M. White, Astrophys. J. 490, 493 (1997)

[5] C. Carignan and S. Beaulieu, Astrophys. J. 347, 760 (1989).

[6] G. Lake, R. A. Schommer and J. H. van Gorkom, Astronomical J. 99, 547 (1990).

[7] M. Jobin and C. Carignan, Astronomical J. 100, 648 (1990).

[8] M. G. Walker and J. Penarrubia, Astrophys. J. 742, 20 (2011).

[9] S. Oh, W. J. G. de Blok, E. Brinks, F. Walter and R.C. Kennicutt Jr., Astrophys. J. 141, 193 (2011).

[10] M. Boylan-Kolchin, J. S. Bullock and M. Kaplinghat, Mon. Not. R. Astron. Soc. 415, L40 (2011).

[11] M. Boylan-Kolchin, J. S. Bullock, and M. Kaplinghat, Mon. Not. R. Astron. Soc. 422, 1203 (2012). 
[12] M. R. Lovell, C. S. Frenk, V. R. Eke, A. Jenkins, L. Gao and T. Theuns, arXiv:1308.1399 [astro-ph.CO].

[13] A.V. Maccio, S. Paduroiu, D. Anderhalden, A. Schneider and B. Moore. MNRAS 424, 1105M (2012); Erratum MNRAS 428, 3715M (2013).

[14] M. Viel, G. D. Becker, J. S. Bolton and M. G. Haehnelt, Phys. Rev. D 88, 043502 (2013); A. Schneider, D. Anderhalden, A. Maccio and J. Diemand, arXiv:1309.5960 [astro-ph.CO].

[15] D. N. Spergel and P. J. Steinhardt, Phys. Rev. Lett. 84, 3760 (2000).

[16] M. Rocha, A.H.G. Peter, J.S. Bullock, M. Kaplinghat, S. Garrison-Kimmel, J. Onorbe and L.A. Moustakas, Mon. Not. Roy. Astron. Soc. 430, 81 (2013).

[17] A. H. G. Peter, M. Rocha, J. S. Bullock and M. Kaplinghat, arXiv:1208.3026 [astro-ph.CO].

[18] A. M. Brooks, M. Kuhlen, A. Zolotov and D. Hooper, Astrophys. J. 765, 22 (2013);

A. M. Brooks and A. Zolotov, arXiv:1207.2468 [astro-ph.CO] (2012).

[19] C. W. Purcell and A. R. Zentner, JCAP 1212, 007 (2012).

[20] E. Hayashi, J. F. Navarro and V. Springel, Mon. Not. Roy. Astron. Soc. 377, 50 (2007).

[21] F. Governato, A. Zolotov, A. Pontzen, C. Christensen, S. H. Oh, A. M. Brooks, T. Quinn and S. Shen, and J. Wadsley, Mon. Not. Roy. Astron. Soc. 422, 1231 (2012).

[22] A. R. Zentner, Phys. Rev. D 80, 063501 (2009).

[23] M. G. Aartsen et al. [IceCube Collaboration], Phys. Rev. Lett. 110, 131302 (2013)

[24] S. W. Randall, M. Markevitch, D. Clowe, A. H. Gonzalez and M. Bradac, Astrophys. J. 679, $1173(2008)$.

[25] J. Zavala, M. Vogelsberger and M.G. Walker, arXiv:1211.6426 [astro-ph.CO] (2012).

[26] A. Gould, Astrophys. J. 321, 571 (1987).

[27] G. Jungman, M. Kamionkowski and K. Griest, Phys. Rept. 267, 195-373 (1996).

[28] Sun Fact Sheet, Nasa: http://nssdc.gsfc.nasa.gov/planetary/factsheet/sunfact.html .

[29] N. Grevesse, M. Asplund, A. Sauval and P. Scott, Astrophys Space Sci. 328, 179 (2010).

[30] P. Salucci, F. Nesti, G. Gentile and C.F. Martins, Astron. Astrophys. 523, A83 (2010).

[31] R. Catena and P. Ullio, JCAP 1008, 004 (2010).

[32] A. Gould, Astrophys. J. 388, 338 (1992).

[33] M. Blennow, J. Edsjö and T. Ohlsson, JCAP 0801, 021 (2008). [arXiv:0709.3898].

[34] J. Beringer et al. [Particle Data Group Collaboration], Phys. Rev. D 86, 010001 (2012).

[35] M. Danninger, Doctoral Thesis in Physics, Stockholm University (2011). (Available at http://su.diva-portal.org/smash/record.jsf?searchId=1\&pid=diva2:621654)

[36] R. Abbasi et al. [IceCube Collaboration], Astropart. Phys. 35, 615 (2012).

[37] D. S. Akerib et al. [LUX Collaboration], arXiv:1310.8214 [astro-ph.CO] (2013).

[38] S. Tulin, H. -B. Yu and K. M. Zurek, Phys. Rev. D 87, 115007 (2013).

[39] Work in progress. 